\title{
Effect of Intermittent Administration of Sustained Release Isosorbide Dinitrate (sr- ISDN) in Rats with Pressure-Overload Heart
}

\author{
Shunsuke SHIMAMURA ${ }^{1}$, Hayato ENDO ${ }^{1}$, Hiromi KUTSUNA ${ }^{1)}$, Masayuki KOBAYASHI ${ }^{1}$, Hidehiro HIRAO ${ }^{1)}$, \\ Miki SHIMIZU' ${ }^{1)}$, Ryou TANAKA ${ }^{1)}$ and Yoshihisa YAMANE ${ }^{1)}$ \\ ${ }^{1)}$ Department of Veterinary Surgery, Faculth of Agriculture, Tokyo University of Agriculture and Technology, 3-5-8 Saiwai-cho, Fuchu- \\ shi, Tokyo 183-8509 Japan
}

(Received 13 September 2005/Accepted 8 November 2005) ABSTRACT. Recent studies have demonstrated the benefits of nitric oxide (NO) on myocardial hypertrophy and myocardial fibrosis. It was
suggested that NO has a protective effect on myocardial cell through the neurohormonal system. This effect serves to highlight the
important role of NO in maintaining the function and form of heart with chronic heart failure. However, there are no known reports
about on the effect of prolonged administration of nitrate on pressure over-load heart. This study was conducted to examine the long-
term effect of oral nitrate therapy in rats with pressure-overloaded heart. An abdominal aorta constricted (AC) model of pressure-over-
loaded heart was created in male Wistar rats. Sustained release isosorbide dinitrate (sr-ISDN) ( $5 \mathrm{mg} / \mathrm{kg}$ once a daily) was administered
to the rats once a daily for 12 weeks. The animals were euthanized during the study period, and the heart was collected and weighed.
Histopathological examination was performed to evaluate the effect of sr-ISDN on myocardial hypertrophy and fibrosis. The ratio of
heart to body weight increased significantly in AC rat and this increase was significantly prevented by sr-ISDN treatment. Histopatho-
logical examination showed significant increase in fibrotic area of AC rat compared to sham rat, this increase was inhibited by sr-ISDN
treatment. Cardiomyocyte transverse diameter was significantly increased in AC rat compared with sham rat, but this increase tended
to decrease by sr-ISDN treatment. In conclusion, intermittent administration with sr-ISDN has mild effect in inhibiting cardiac hyper-
trophy and marked effect in inhibiting fibrosis due to pressure-overload.
KEY wORDS: aortic constrict model, cardiac fibrosis, cardiac hypertrophy, isosorbide dinitrate, rat.

Isosorbide dinitrate (ISDN) belongs to a group of compounds called organic nitrates. Organic nitrates are vasodilators which induce vascular smooth muscle relaxation through a pathway involving biotransformation from nitrate to NO $[3,26]$. Nitrates works in all vascular smooth muscles, especially in venous and coronal vessels, in decreasing pre-load and preventing ischemic heart disease $[12,22]$. For this reason, nitrates have been used in the treatment of angina pectoris $[1,11]$, ischemic heart disease and chronic heart failure in humans $[5,6,20,25]$. The effect of nitrates are observed not only in relation to neurohormonal system but also to hemodynamics. Several researchers have demonstrated the regulatory role of NO in modulating extracellular matrix. Hou et al. [7] had shown that chronic NO synthase inhibition induces cardiac fibrosis in rat. They suggested that NO work as a regulator in fibroblast proliferation in cardiomyocytes. NO was also shown to be an important agent in modulating the synthesis of extracellular matrix proteins $[16,30]$. The antiproliferative effect of NO in extracellular matrix protein depresses cardiac remodeling due to pressure-overload or myocardial infarction [14]. These reports suggest direct effect of NO on cardiomyocyte protection. Histopathological changes in pressure-overload heart are characterized by concentric hypertrophy with myocardial hypertrophy and fibrosis due to proliferating fibroblasts in the interstitium [18]. Organic changes in the heart, including myocardial fibrosis and myocardial hypertrophy, result in cardiac dysfunction. The inhibitory effect of NO on these changes is thought to be an important factor in heart failure therapy. Previous experiments in vivo have shown that NO suppresses myocardial fibrosis and myocyte hypertrophy [21, 27]. Furthermore, NO has inhibitory effect on cardiac remodeling of angiotensin converting enzyme inhibitor and angiotensin receptor blocker mediated by increasing endogenous NO production intervening kinin [15, 17]. Further studies indicate useful effects of NO on morphological changes in the heart due to pressure-overload. However, there is no known report on the efficacy of long-term administration of nitrate on pressure-overload heart.

In this study, the effect of prolonged administration of nitrate on rats with cardiac pressure-overload was investigated using an aortic constricted model (AC). Intermittent administration of sustained-release ISDN (sr-ISDN), at a dose rate of $5 \mathrm{mg} / \mathrm{kg}$ once a day, was used, because previous studies have shown that an intermittent dosing regimen, including a nitrate-free interval of 10 to $12 \mathrm{hr}$, is an effective therapeutic approach for preventing the development of nitrate tolerance. Myocardial fibrosis and myocardial hypertrophy was examined through histopathological evaluation in rats with pressure-overload treated with sr-ISDN.

\section{MATERIALS AND METHODS}

Animals: Twenty-one male Wistar rats (Japan Saitama Experimental Animal Supply, Saitama, Japan), weighing approximately $200 \mathrm{~g}$ each, were used in the study. The rats were housed under standard enviromental conditions and maintained on commercial rat feed and given tap water $a d$ 
libitum.

$I S D N$ : The sr-ISDN was purchased from Eisai Company (Japan). The sr-ISDN was dissolved in distilled water $(0.5$ $\mathrm{m} l$ ) and given orally.

Methods: The protocol used in the study has been approved by the Laboratory Animal Care Committee of the Tokyo University of Agriculture and Technology.

Experimental model: An abdominal aorta constricted (AC) model of pressure-over loaded heart was created in rats according to the procedures described by Morkin et al. [23]. After anesthesia was induced with an intraperitoneal injection of sodium pentobarbital (NEMBUTAL Injection : Dainippon Pharmaceutical Co., Ltd. Japan) a ventral abdominal laparotomy was performed to expose the aorta. In order to assure a reasonably uniform degree of constriction of abdominal aorta below the renal artery, the constricting tie was placed over a 21-gauge needle which was then removed, leaving a constriction equal to the diameter of the needle. The abdominal musculature and skin incision were closed using standard techniques. Sham-operated animals were subjected to the same surgical procedure without creation of AC. The rats were allowed a seven-day recovery period to adjust before the treatment was started.

Experimental protocol: The rats were randomly divided into four groups consisting of 7 animals for each group, as follows:1)AC rats given oral sr-ISDN at $5 \mathrm{mg} / \mathrm{kg}$ (AC-srISDN); 2) AC rats given oral distilled water as placebo (ACPlacebo); 3) sham-operated rats given oral distilled water as placebo (Sham-Placebo). Distilled water and sr-ISDN were administered via gastric gavage once a day for 12 weeks. To avoid the initial stress of surgery, the treatments started 7 days after $\mathrm{AC}$ and sham operations and continued for the entire duration of the experiment. At the end of 12 weeks, the rats were anesthetized with sodium pentobarbital and cardiac arrest was induced in diastole by intravenous injection of $20 \% \mathrm{KCl}$ solution $(0.2 \mathrm{ml} / 100 \mathrm{~g}$ body weight). The rats were then necropsied and the hearts were collected and weighed.

Histopathological analysis: The hearts were fixed in 10\% formalin, dehydrated with ethanol, embedded in paraffin, and sectioned at a thickeness of $3 \mu \mathrm{m}$. Histopathological examination was performed in a blind fashion by 2 observers for 3 independent samples from each subject. To determine myocardial hypertrophy, the shortest transverse diameter was measured in 100 nucleated transverse section of the cardiomyocytes of the left ventricle (LV) myocardium stained with hematoxylin-eosin, using a microscope (OLYMPAS KS-630 KS: OLYMPAS, Japan) connected to a computer with image analysis software (Mac-Scope, Ver.2.6, Mitani, Tokyo, Japan) [13]. The cardiomyocytes were selected which cells showed the spindle-shape, including the elliptical nucleus in transverse section. Usually, 100 cells that satisfied the selection criteria could be found per one observation field at $200 \times$ magnification, and 5 fields were randomly selected per sample slide. Finally, the average of the diameter was calculated as group mean. To determine myocardial fibrosis, the volume percent collagen in each field was measured in picric acid Sirius red stained sections of the LV myocardium, using a microscope connected to a computer with an image analysis software, as previously described [34]. A minimum of 10 fields from each of four LV sections (inner, mid, outer) was examined using the $200 \times$ magnification. To assess changes in perivascular fibrosis, the ratio of the area of pericascular fibrosis with vessel lumen area was obtained. Collagen volume percent for each animal was expressed as the average of all fields examined.

Statistical analysis: Data were expressed as means \pm S.E. The differences between the groups were evaluated using one-way analysis of variance (ANOVA) followed by the Tukey-Kramer test. Temporal differences among groups were evaluated using repeated measure ANOVA followed by the Tukey-Kramer test. The level of significant differences was set at $\mathrm{p}<0.05$.

\section{RESULTS}

Effect of sr-ISDN on heart weight: The heart weight to body weight ratio was calculated after 12 weeks of treatment either with sr-ISDN or placebo. These results are shown in Fig. 1. The ratio in AC-Placebo group significantly increased compared to the Sham-Placebo group $(p<0.05)$. The increase in AC-sr-ISDN group was lower than with AC-Placebo group $(\mathrm{p}<0.05)$.

Effect of sr-ISDN on cardiomyocyte: Quantitative distribution of the cardiomyocyte transverse diameter was shown in Fig. 2. The cardiomyocyte transverse diameter in ACPlacebo and AC-sr-ISDN groups increased significantly compared with the sham-Placebo group $(p<0.05)$. The diameter in AC-sr-ISDN group was lesser than that of the AC-Placebo group, although no significant difference was

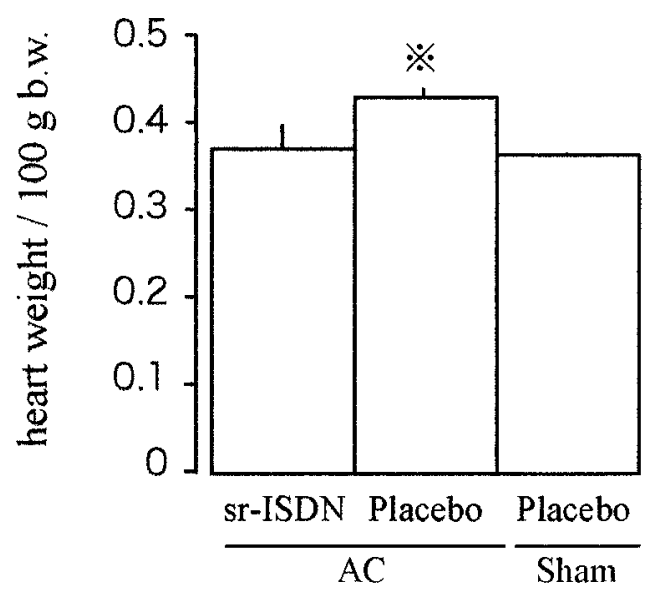

Fig. 1. Effect of oral intermittent administration of srISDN on heart weight in AC rats. The animals were euthanized at the end of experimental period, and the heart was collected and weighed. The heart weight is expressed as the ratio of heart weight to body weight. Data are expressed as means \pm S.E. $※ p<0.05$ vs Sham-Placebo group. 


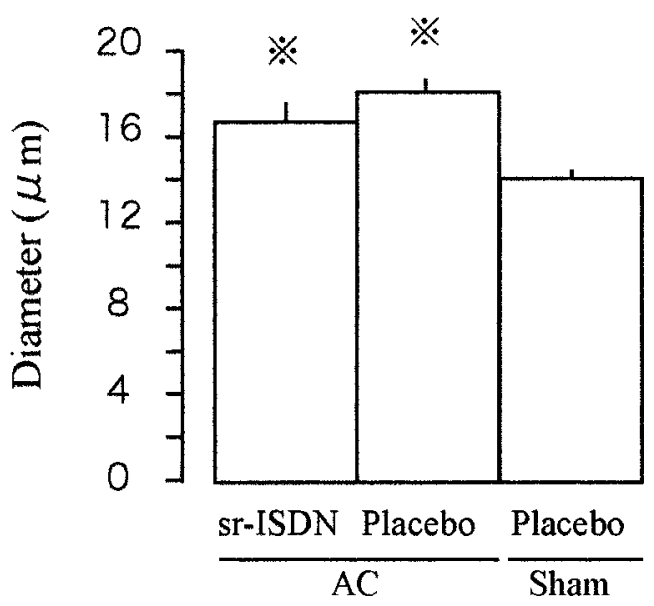

Fig. 2. Effect of oral administration of sr-ISDN on myocardial hypertrophy in AC rats. To determine myocardial hypertrophy, the transverse diameter $(\mu \mathrm{m})$ of the left ventriclar cardiomyocytes was measured. Data are expressed as means \pm S.E. $※ p<0.05$ vs Sham-Placebo group.

observed between the two groups $(\mathrm{p}>0.05)$. Interstitial fibrosis and perivascular fibrosis of the left ventricle were seen in the samples stained using picric acid Sirius red stain (Fig. 3). The quantitative distribution of the volume percentage of interstitial fibrosis and perivascular fibrosis was shown in Fig. 4. Both perivascular and interstitial collagen were increased significantly in AC-Placebo group compared with Sham groups $(\mathrm{p}<0.05)$. The volume of both perivascular and interstitial collagen in the AC-sr-ISDN group was significantly lower than that of the AC-Placebo group $(\mathrm{p}<0.05)$.

\section{DISCUSSION}

The present study demonstrated that mild inhibition of myocardial hypertrophy and significant inhibition of myocardial fibrosis were presented by prolonged intermittent administration of sr-ISDN. Nitrates have been shown to induce immediate tolerance in previous studies conducted in vivo and in vitro $[8,9,19,28]$, although the mechanism of tolerance induction through nitrate administration has been unclear until the present time. However, it has been demonstrated that the intermittent administration of nitrates with a drug-free time of 10 to 12 hours can prevent development of tolerance $[10,24]$. In this study, sr-ISDN was administered intermittently with a drug-free time 12 hours of once a day treatment.

After-load of the left ventricle was increased due to abdominal aortic constriction in AC model rats. Increase in after-load made augments myocardial rigidity, resulting in constricted hypertrophy characterized by increasing myocardial transverse diameter. Interstitial myocardial fibrosis due to fibroblast activation occurred with progression of constricted hypertrophy. The AC rat, in the present study,
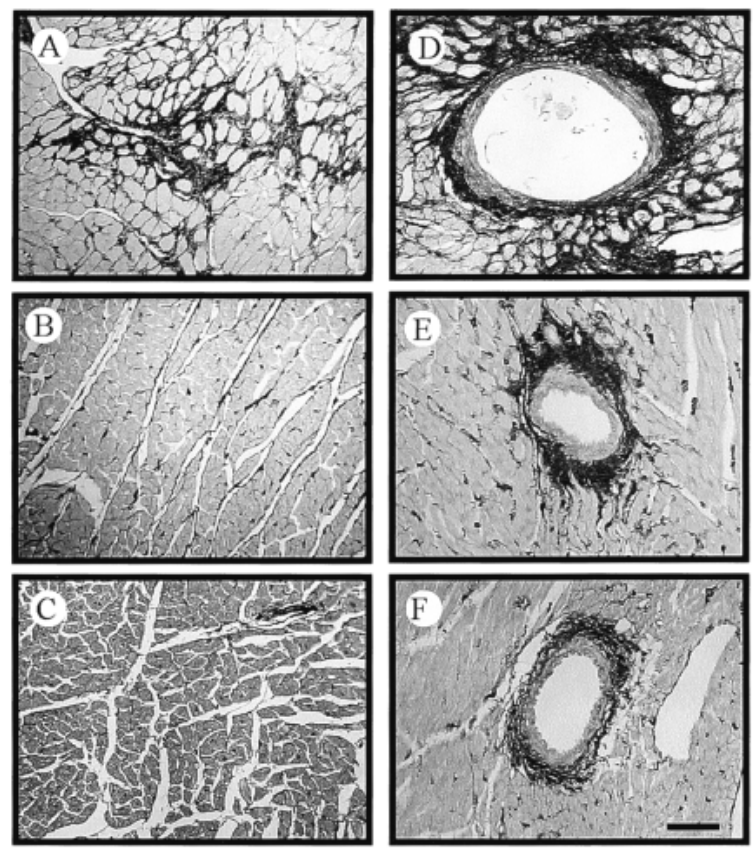

Fig. 3. Interstitial fibrosis (A, B, C) and perivascular fibrosis (D, E, F, G) in AC model rats (picric acid Sirius stain). Scale bar $=50 \mu \mathrm{m}$. A and D: AC-Placebo, B and E: AC-sr-ISDN, $\mathrm{C}$ and F: Sham-Placebo.
A

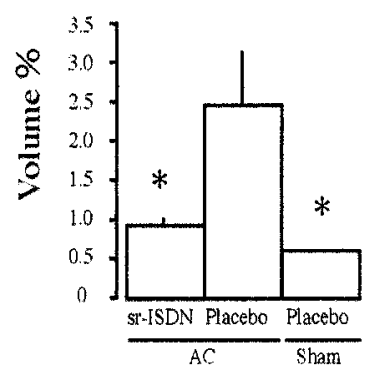

B

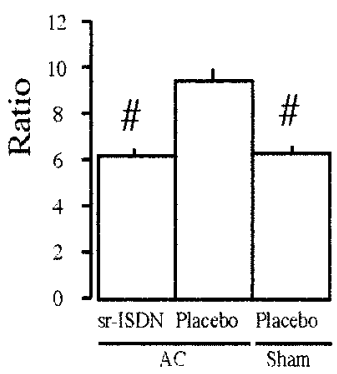

Fig. 4. Effect of oral administration of sr-ISDN on area of fibrosis in AC rats. (A) Perivascular fibrosis area is expressed by the ratio of area of perivascular fibrosis to vascular lumen area. (B) myocardial interstitial fibrosis in expressed as volume percentage. ${ }^{*}$ p $<0.05$ vs AC-Placebo group. \# $\mathrm{p}<0.05$ vs AC-Placebo group.

showed an increased heart weight. Furthermore, myocardial transverse diameter increased with development of both myocardial and perivascular fibrosis. These results showed that constricted hypertrophy due to pressure-overload can result in the development of myocardial hypertrophy and myocardial fibrosis.

The inhibitory effect of intermittent administration of srISDN on myocardial hypertrophy was demonstrated in the $\mathrm{AC}$ rat. Myocardial transverse diameter in both AC-srISDN and AC-Placebo groups increased significantly com- 
pared to the Sham-Placebo group, but the increase in the AC-sr-ISDN group was smaller than the AC-Placebo group. Furthermore, an increase in the ratio of heart weight to body weight due to constricted hypertrophy was inhibited significantly in sr-ISDN administrated animals. These results suggest that sr-ISDN inhibits myocardial hypertrophy. Previous studies have shown that chronic administration of L-arginine, the precursor of $\mathrm{NO}$, attenuated cardiac hypertrophy in spontaneously hypertensive rats [21] and $\mathrm{NO} /$ cGMP was shown to be a negative regulator of cardiomyocyte hypertrophy without changing blood pressure [33]. These experiments suggest direct inhibitory effect of $\mathrm{NO}$ on myocardial hypertrophy, which is consistent with the results obtained in the present study.

However, significant difference in myocardial transverse diameter was not observed between AC-sr-ISDN and ACPlacebo groups, although the difference in heart weight was significant. This is the reason why myocardial transverse diameter was slightly different in one dimension augmented in three dimensions. It is also possible that increase in myocardial fibrotic area strongly affected heart weight. Whereas L-arginine was used in previous studies in vivo [21], it was suggested that through oral administration of srISDN in the present study, the animal was not able to obtain sufficient dose of nitric oxide to significant suppress myocardial hypertrophy. A significant finding in this study is the suppression of myocardial fibrosis in the $\mathrm{AC}$ rat through intermittent administration of sr-ISDN. Essential changes in myocardial fibrosis in pressure-overload heart were observed in perivascular and interstitial fibrosis. At first, myocardial fibrosis occurred around the coronal vessels, and then progressed in the interstitial tissues $[4,29,32]$. Intermittent administration of sr-ISDN significantly suppressed in both the coronal vessels and interstitial tissues. These results suggest marked anti-myocardial fibrosis effect of $\mathrm{NO}$.

The anti-myocardial fibrosis effect of NO has been demonstrated in many experiments in vivo and in vitro $[2,14$, 16], and was consistent with the result obtained in the present study. Previous studies and the results of the present study suggest the possibility that sr-ISDN administration improve the diastolic capacity of the left ventricule of pressure-overload heart resulting in the anti-myocardial fibrosis effect. Excessive deposition of fibroblast in the myocardial tissue depresses the rigidity of the heart, leading to decrease in the diastolic capacity. Finally, cardiac dysfunction causes regression of the systolic capacity [31]. Therefore, inhibition of myocardial fibrosis maintains the rigidity of the heart, leading to improve cardiac function. Our observation that the anti-myocardial fibrosis effect of sr-ISDN worked in maintenance of the stiffness of $\mathrm{AC}$ rat was showed the long-term effectiveness of sr-ISDN administration to pressure-overload heart. In conclusion, the results of the study suggest that sr-ISDN administration affects pressure-overload heart by inhibition of myocardial fibrosis and myocardial hypertrophy.

\section{REFERENCES}

1. Aronow, S. 1980. Sustained hemodynamic and antianginal effects of high dose oral isosorbide dinitrate. Nouv. Presse. Med. 9: 2483-2486.

2. Bledsoe, G., Chao, L. and Chao, J. 2003. Kallikrein gene delivery attenuates cardiac remodeling and promotes neovascularization in spontaneously hypertensive rats. Am. J. Physiol. Heart Circ. Physiol. 285: H1479-H1488.

3. Bomber, J.W. and De Tullio, P.L. 1995. Oral nitrate preparations: an update. Am. Fam. Physician. 52: 2331-2337.

4. Cheitlin, M.D., Robinowitz, M., McAllister, H., Hoffman, J.I., Bharati, S. and Lev, M. 1980. The distribution of fibrosis in the left ventricle in congenital aortic stenosis and coarctation of the aorta. Circulation 62: 823-830.

5. Cohn, J.N., Archibald, D.G., Ziesche, S., Franciosa, J.A., Harston, W.E., Tristani, F.E., Dunkman, W.B., Jacobs, W., Francis, G.S., Flohr, K.H., et al. 1986. Effect of vasodilator therapy on mortality in chronic congestive heart failure. Results of a Veterans Administration Cooperative Study. New Engl. J. Med. 314: 1547-1552.

6. Cohn, J.N. 1992. Mechanisms of action and efficacy of nitrates in heart failure. Am. J. Cardiol. 70: 88B-92B.

7. Hou, J., Kato, H., Cohen, R.A., Chobanian, A.V. and Brecher, P. 1995. Angiotensin II-induced cardiac fibrosis in the rat is increased by chronic inhibition of nitric oxide synthase. J. Clin. Invest. 96: 2469-2477.

8. Elkayam, U., Kulick, D., McIntosh, N., Roth, A., Hsueh, W. and Rahimtoola, S.H. 1987. Incidence of early tolerance to hemodynamic effects of continuous infusion of nitroglycerin in patients with coronary artery disease and heart failure. Circulation 76: 577-584.

9. Elkayam, U. 1991. Tolerance to organic nitrates: evidence, mechanisms, clinical relevance, and strategies for prevention. Ann. Intern. Med. 114: 667-677.

10. Ishikawa, K., Yamamoto, T., Kanamasa, K., Hayashi, T., Takenaka, T., Kimura, A., Miyataka, M., Yabushita, H. and Kitayama, K. 2000. Intermittent nitrate therapy for prior myocardial infaraction does not induce rebound angina nor reduce cardiac events. Intern. Med. 39: 1020-1026.

11. Jugdutt, B.I. and Warnica, J.W. 1988. Intravenous nitroglycerin therapy to limit myocardial infarct size, expansion, and complications. Effect of timing, dosage, and infarct location. Circulation 78: 906-919.

12. Jugdutt, B.I. and Khan, M.I. 1994. Effect of prolonged nitrate therapy on left ventricular remodeling after canine acute myocardial infarction. Circulation 89: 2297-2307.

13. Kai, H., Muraishi, A., Sugiu, Y., Nishi, H., Seki, Y., Kuwahara, F., Kimura, A., Kato, H. and Imaizumi, T. 1998. Expression of proto-oncogenes and gene mutation of sarcomeric proteins in patients with hypertrophic cardiomyopathy. Circ. Res. 83: 594-601.

14. Kim, N.N., Villegas, S., Summerour, S.R. and Villarreal, F.J. 1999. Regulation of cardiac fibroblast extracellular matrix production by bradykinin and nitric oxide. J. Mol. Cell. Cardiol. 31: 457-466.

15. Kobayashi, N., Higashi, T., Hara, K., Shirataki, H. and Matsuoka, H. 1999. Effects of imidapril on NOS expression and myocardial remodelling in failing heart of Dahl salt-sensitive hypertensive rats. Cardiovasc. Res. 44: 518-526.

16. Kolpakov, V., Gordon, D. and Kulik, T.J. 1995. Nitric oxidegenerating compounds inhibit total protein and collagen synthesis in cultured vascular smooth muscle cells. Circ. Res. 6: 
305-309.

17. Kurisu, S., Ozono, R., Oshima, T., Kambe, M., Ishida, T., Sugino, H., Matsuura, H., Chayama, K., Teranishi, Y., Iba, O., Amano, K. and Matsubara, H. 2003. Cardiac angiotensin II type 2 receptor activates the kinin/NO system and inhibits fibrosis. Hypertension 41: 99-107.

18. Kuwahara, F., Kai, H., Tokuda, K., Kai, M., Takeshita, A., Egashira, K. and Imaizumi, T. 2002. Transforming growth factor-beta function blocking prevents myocardial fibrosis and diastolic dysfunction in pressure-overloaded rats. Circulation 106: $130-135$.

19. Leier, C.V. Nitrate tolerance. 1985. Am. Heart J. 110: 224232.

20. Massie, B., Chatterjee, K., Werner, J., Greenberg, B., Hart, R. and Parmley, W.W. 1977. Hemodynamic advantage of combined administration of hydralazine orally and nitrates nonparenterally in the vasodilator therapy of chronic heart failure. Am. J. Cardiol. 40: 794-801.

21. Matsuoka, H., Nakata, M., Kohno, K., Koga, Y., Nomura, G., Toshima, H. and Imaizumi, T. 1996. Chronic L-arginine administration attenuates cardiac hypertrophy in spontaneously hypertensive rats. Hypertension 27: 14-18.

22. McDonald, K.M., Francis, G.S., Matthews, J., Hunter, D. and Cohn, J.N. 1993. Long-term oral nitrate therapy prevents chronic ventricular remodeling in the dog. J. Am. Coll. Cardiol. 21: 514-522.

23. Morkin, E. and Ashford, T.P. 1968. Myocardial DNA synthesis in experimental cardiac hypertrophy. Am. J. Physiol. 215: 1409-1413.

24. Muller, S., Laber, U., Mullenheim, J., Meyer, W. and Kojda, G. 2003. Preserved endothelial function after long-term eccentric isosorbide mononitrate despite moderate nitrate tolerance. J. Am. Coll. Cardiol. 41: 1994-2000.

25. Parmley, W.W. 1985. Role of isosorbide dinitrate in management of chronic congestive heart failure. Am. Heart. J. 110: 264-268.
26. Palmer, R.M., Ferrige, A.G. and Moncada, S. 1987. Nitric oxide release accounts for the biological activity of endothelium-derived relaxing factor. Nature (Lond.) 327: 524-526.

27. Rossi, M.A., Ramos, S.G. and Prado, C.M. 2003. Chronic inhibition of nitric oxide synthase induces hypertension and cardiomyocyte mitochondrial and myocardial collagen remodelling in the absence of hypertrophy. J. Hypertens. 21: 993-1001.

28. Rudolph, W., Dirschinger, J., Reiniger, G., Beyerle, A. and Hall, D. 1988. When does nitrate tolerance develop? What dosages and which intervals are necessary to ensure maintained effectiveness? Eur. Heart J. 9: 63-72.

29. Schwarz, F., Flameng, W., Schaper, J., Langebartels, F., Sesto, M., Hehrlein, F. and Schlepper, M. 1978. Myocardial structure and function in patients with aortic valve disease and their relation to postoperative results. Am. J. Cardiol. 41: 661-669.

30. Trachtman, H., Futterweit, S. and Singhal, P. 1995. Nitric oxide modulates the synthesis of extracellular matrix proteins in cultured rat mesangial cells. Biochem. Biophys. Res. Commun. 207: 120-125.

31. Weber, K.T. and Brilla, C.G. 1991. Pathological hypertrophy and cardiac interstitium. Fibrosis and renin-angiotensin-aldosterone system. Circulation 83: 1849-1865.

32. Weber, K.T., Brilla, C.G., Campbell, S.E., Zhou, G., Matsubara, L. and Guarda, E. 1992. Pathologic hypertrophy with fibrosis: the structural basis for myocardial failure. Blood Press. 1: 75-85.

33. Wollert, K.C., Fiedler, B., Gambaryan, S., Smolenski, A., Heineke, J., Butt, E., Trautwein, C., Lohmann, S.M. and Drexler, H. 2002. Gene transfer of cGMP-dependent protein kinase I enhances the antihypertrophic effects of nitric oxide in cardiomyocytes. Hypertension 39: 87-92.

34. Yang, G., Meguro, T., Hong, C., Asai, K., Takagi, G., Karoor, V.L., Sadoshima, J., Vatner, D.E., Bishop, S.P. and Vatner, S.F. 2001. Cyclosporine reduces left ventricular mass with chronic aortic banding in mice, which could be due to apoptosis and fibrosis. J. Mol. Cell. Cardiol. 33: 1505-1514. 